\title{
Museología 4.0. Un ejemplo real
}

\section{Museology 4.0. Real-world example}

\author{
Alexandre González Rivas ${ }^{\mathrm{a}}$, Xoel Vázquez Dios ${ }^{\mathrm{b}}$ \\ ${ }^{a}$ Xoia Extending Reality. Moaña. e: Alexandre.gonzalez@xoia.es ${ }^{\text {b }}$ Xoia Extending Reality. A \\ Coruña. e: Xoel.vazquez@xoia.es
}

\begin{abstract}
Resumen
En la presente comunicación se va a abordar la aplicación práctica en casos de uso reales de la Realidad Virtual, Aumentada y otras tecnologías interactivas e inmersivas de vanguardia, tanto en el contexto de Museo 4.0 como en espacios de interés patrimonial como pueden ser yacimientos, ciudades monumentales o rutas jacobeas. Se explicará, con ejemplos reales y recientes como Xoia Extending Reality afronta las nuevas necesidades tecnológicas del Museo 4.0, con el objetivo de mejorar la interpretación, estudio, divulgación y promoción de nuestra Historia y Patrimonio mediante el empleo de las NTIC, especialmente Realidad Aumentada y Virtual, centrándose en la experiencia de usuario y en la calidad de lo divulgado. Para ello se describen casos de éxito desarrollados por Xoia Extending Reality como la exposición coordinada por la Xunta de Galicia, "Galicia un relato no mundo", la experiencia turística de Realidad Aumentada "Viaxando polo Pontedeume medieval", desarrollada para el ayuntamiento de Pontedeume en un contexto de promoción del camino de Santiago, entre otros proyectos de puesta en valor patrimonial, cultural y turística, desarrollados con ayuntamientos, universidades y/o otras instituciones, vinculando de forma innovadora el turismo, tecnología y cultura con la finalidad de divulgar de forma novedosa y potenciando el aprendizaje, logrando llegar a un público cada vez más digital. Por último se citará ejemplos prácticos de soluciones tecnológicas aplicadas al contexto del COVID -19.
\end{abstract}

Palabras clave: realidad virtual, realidad aumentada, Roía Extending Reality, museo 4.0, virtualización del Patrimonio

\footnotetext{
Abstract

This document will address the practical application in real use cases of Virtual Reality, Augmented Reality and other avant-garde interactive and immersive technologies, both in the context of Museum 4.0 and in spaces of heritage interest such as sites, monumental cities or xacobeas routes. It will be
} 
explained, with real and recent examples, how Xoia Extending Reality addresses the new technological needs of the Museum 4.0, with the aim of improving the interpretation, study, dissemination and promotion of our History and Heritage through the use of the new ICT, especially Augmented Reality and Virtual Reality, focusing on the user experience and the quality of what is disclosed. For this, success stories developed by Xoia are described, such as the exhibition coordinated by the "Xunta de Galicia", "Galicia, un relato no mundo", the augmented reality tourist experience "Viaxando polo Pontedeume medieval", developed for the city council of Pontedeume in a context of promotion of the Saint James Way, among other projects to enhance heritage, culture and tourism, developed with city councils, universities and / or other institutions, innovatively linking tourism, technology and culture in order to disseminate in an innovative way and enhancing learning, reaching an increasingly digital audience. Finally, practical examples of technological solutions applied to the context of COVID -19 will be cited.

Keywords: virtual reality, augmented reality, Xoia Extending Reality, museum 4.0, virtual heritage. 


\section{Introducción}

La aplicación a nivel mundial de la visualización asistida por ordenador en el campo del Patrimonio, el espectacular crecimiento del turismo cultural y los increíbles avances tecnológicos desarrollados en los últimos años, han propiciado la elaboración y ejecución de un sin fin de proyectos encaminados a investigar, preservar, interpretar y presentar distintos elementos del patrimonio, presente o no, a partir de las nuevas tecnologías aplicadas a los museos y al Patrimonio material e inmaterial en su conjunto.

La explosión tecnológica del momento y su aplicación en todos los ámbitos de nuestras vidas, refleja la necesidad de mejorar o actualizar la museología tradicional, combinando lo tradicional y la innovación como herramientas de divulgación y aprendizaje. Este documento pretende mostrar varios de los múltiples usos de las Nuevas Tecnologías de Información y Comunicación (NTIC) vinculadas con la interpretación, promoción y puesta en valor turística de recursos culturales, destacando la Realidad Virtual (RV) y Realidad Aumentada (RA), que ha realizado Xoia Extending Reality con el objetivo de satisfacer la demanda tecnológica que necesita la museología 4.0 y las diferentes ramas que la componen, ya que, actualmente cualquier destino que pretenda ser competitivo debe actualizar continuamente toda aquella información que pueda ser interés para el visitante.

El turista cuya principal motivación es la cultura, se caracteriza por requerir gran cantidad de información y ser consumidor de Tics. Los usuarios se han formado en usuarios 2.0 caracterizados por estar altamente conectados y por ser generadores de información y, sobre todo de opinión en redes sociales, blogs, etc., siendo claves en los sistemas de reputación online. Por lo tanto, el fuerte desarrollo experimentado por el turismo cultural en los últimos años, se enmarca en los cambios acaecidos en los destinos turísticos ante los procesos de diversificación y especialización de la demanda, que obligan a estos espacios a una búsqueda constante de singularización y diferenciación de sus productos que atiendan este consumo individualizado (Granja, 2015) .

La aplicación de tecnologías 3D es un tema recurrente que se lleva estudiando hace ya décadas. No obstante, en la actualidad es cuando realmente se está en disposición de llevarla a la práctica para el turista. La aparición de Smartphone, tablets y phablets con alta conectividad y gran capacidad de procesamiento gráfico ha hecho posible su uso en este campo, logrando que la tecnología 3D sea un marco de referencia para lo que se conoce como aplicaciones de realidad virtual y realidad aumentada (José Luís Caro, 2013).

Estamos en un momento idóneo para el desarrollo de experiencias digitales en museos, y en general de las nuevas tecnologías aplicadas a los ámbitos del patrimonio, turismo y cultura, que se está desarrollando en diversas parcelas: gestión del patrimonio cultural y las aplicaciones informáticas asociadas, aplicaciones web para la difusión y registro del patrimonio (incluyéndose las redes sociales), análisis geoespacial, registro espacial, temas relacionados con la digitalización, realidad virtual, realidad aumentada, etc. El conjunto de estas parcelas alcanza un grado tecnológico que nos permitirá avanzar en la aplicación de 
nuevos objetivos (antes inalcanzables) para el beneficio del turismo, la cultura y el Patrimonio.

En los últimos años la realidad virtual y aumentada está consiguiendo asentarse en un nuevo prototipo de museo: el inmersivo. Estas tecnologías tienen la capacidad de insertar objetos virtuales en el espacio real y se ha convertido en una herramienta muy útil para presentar determinados contenidos bajo las premisas de entretenimiento y educación, generando una demanda tecnológica que necesita de personal cualificado y multidisciplinar. Xoia Extending Reality pretende satisfacer esa demanda usando la última tecnología de vanguardia, siguiendo una metodología heredada de experiencias en diferentes ámbitos de actuación, con el fin de transmitir conocimiento, bajo el relieve de las nuevas tendencias museográficas, logrando crear contenidos en la que los visitante no solo actúan como meros observadores pasivos, sino que interactúan con los contenidos que se presentan bajo un enfoque lúdico-racional.

\section{Objetivos}

Con carácter general, se pretende mostrar en este artículo diferentes proyectos en los que se ha sumergido Xoia Extending Reality a lo largo de la geografía nacional y en diferentes ámbitos tecnológicos centrándose, de forma más específica, en los proyectos que se realizaron en museos o galerías de arte, con el objetivo de mostrar diferentes posibilidades del uso de realidad virtual y aumentada, así como el empleo de diferentes NTICs. También como se ha utilizado esta tecnología en espacios reales aportando soluciones tecnológicas que mejoran la experiencia museística. Por otra parte se mostrará cuáles son las herramientas y la metodología a seguir para poder lograr los objetivos educativos exigidos tanto por clientes como por el público.

Finalmente se expondrán en el presente documento casos concretos en las experiencias que Xoia Extending Reality ha realizado fuera del espacio museístico tradicional, aplicando la tecnología de realidad aumentada en rutas turísticas al aire libre o creando en realidad virtual el propio espacio museístico digitalizado. Además, se expondrán soluciones prácticas para los museos 4.0 en el entorno del COVID-19 y cuáles son las soluciones aplicadas por Xoia Extending Reality en casos reales.

El papel de Xoia Extending Reality en estas experiencias, así como sus objetivos, ha sido el desenvolvimiento de una infraestructura tecnológica de vanguardia, garantizar el ecoeficiencia del destino mejorando la conexión entre destinos, personas y servicios, además de crear conexiones emocionales entre el visitante y el destino facilitando y mejorando la accesibilidad y la interpretación del patrimonio. Para poder comprender como se han logrado estos objetivos, debemos profundizar en cómo se tuvo que afrontar cada uno de estos proyectos y cuáles son los recursos necesarios para llevarlo a cabo. 


\section{Desarrollo de la innovación}

Desde un primer momento se muestra la necesidad de un equipo multidisciplinar: personas especialistas en cada uno de los diferentes ámbitos que necesitan los proyectos de AR/VR en museos. Hablamos de especialistas de profesiones ya clásicas como ingenieros informáticos o ilustradores, o de profesiones que aparecen a la par de la tecnología, como el diseñador UI/UX, que profundiza en el aspecto visual de la interfaz y de garantizar que la navegación y acciones que realiza el usuario tengan coherencia, graduados en diseño y desarrollo de videojuegos, o virtualizadores del Patrimonio: personas que proceden de diferentes ramas de humanidades: historiadores, historiadores del arte, arquitectos, etc., pero con un perfil informático desarrollado entorno a la virtualización y digitalización del Patrimonio. Esta serie de factores mejoran el resultado final ya que aportan información desde diferentes puntos de vista y aportan soluciones en diferentes ramas que necesitan las aplicaciones de RA y RV, a la vez y mejoran las habilidades sociales tanto en el propio grupo como con los diferentes grupos que componen la acción museística de grandes exposiciones.

A la par, como se ha mencionado anteriormente, es necesario conocer los últimos avances tecnológicos de cada materia para poder subministrar las herramientas adecuadas para cada proyecto como los tipos y funciones de las diferentes gafas de RV y su aplicación. De los softwares necesarios para la producción de cada una de las experiencias o de como transmitir el contenido en cuestión tal y como se pretende antes de iniciar cada proyecto.

Esta serie de factores, han sido superados gratamente por Xoia Extending Reality, logrando objetivos y casos de éxito en casos reales de museología 4.0, como puede ser la exposición "Galicia: Un relato no mundo" en La ciudad de la Cultura en Santiago de Compostela o “ARTguide”, creada para la Fundación María José Joven, logrando transmitir conocimiento, sensaciones, valores y sobre todo tecnología.

\subsection{Exposición "Galicia un relato no mundo"}

Galicia, un relato en el mundo explora la interacción entre el territorio histórico gallego y el resto del mundo en un recorrido desde la prehistoria hasta la actualidad. Concebida como la primera de tres grandes exposiciones de la Xunta con motivo del Xacobeo 2021, se centra en las relaciones internacionales de Galicia y su presencia en el mundo.

La exposición agrupó numerosas piezas de alto valor simbólico para la historia y la cultura de Galicia, conservadas en prestigiosas colecciones museísticas de todo el mundo, como la Cambridge University- Parker Library, las Bibliotecas Bodleian de la Universidad de Oxford, el Museo del Prado, la Biblioteca Apostólica Vaticana, la Biblioteca de Arezzo en Italia o el Museu Nacional de Arqueologia de Portugal. En muchos casos, llegaron a Galicia por vez primera para ser exhibidas en esta muestra, comisariada por el periodista, profesor y divulgador Manuel Gago Mariño. En una exposición con gran apuesta tecnológica y liderada por Xoia Extending Reality, ha sido varias las novedades tecnológicas que se han presentado en $A$ cidade da Cultura. 


\subsubsection{Realidad Virtual: Os Adeuses}

La primera ha sido la experiencia "Os Adeuses", una experiencia inmersiva de realidad virtual, en la que los usuarios son los protagonistas. Gracias a la realidad virtual, los visitantes de "Galicia un Relato no Mundo" se ponen en la piel de los y las emigrantes que partieron hacia América, comprendiendo de un modo hasta ahora no explorado la intensidad de aquellas transoceánicas que cambiaron sus vidas, en este caso cargados de una mochila y gafas de realidad virtual (Fig. 1).

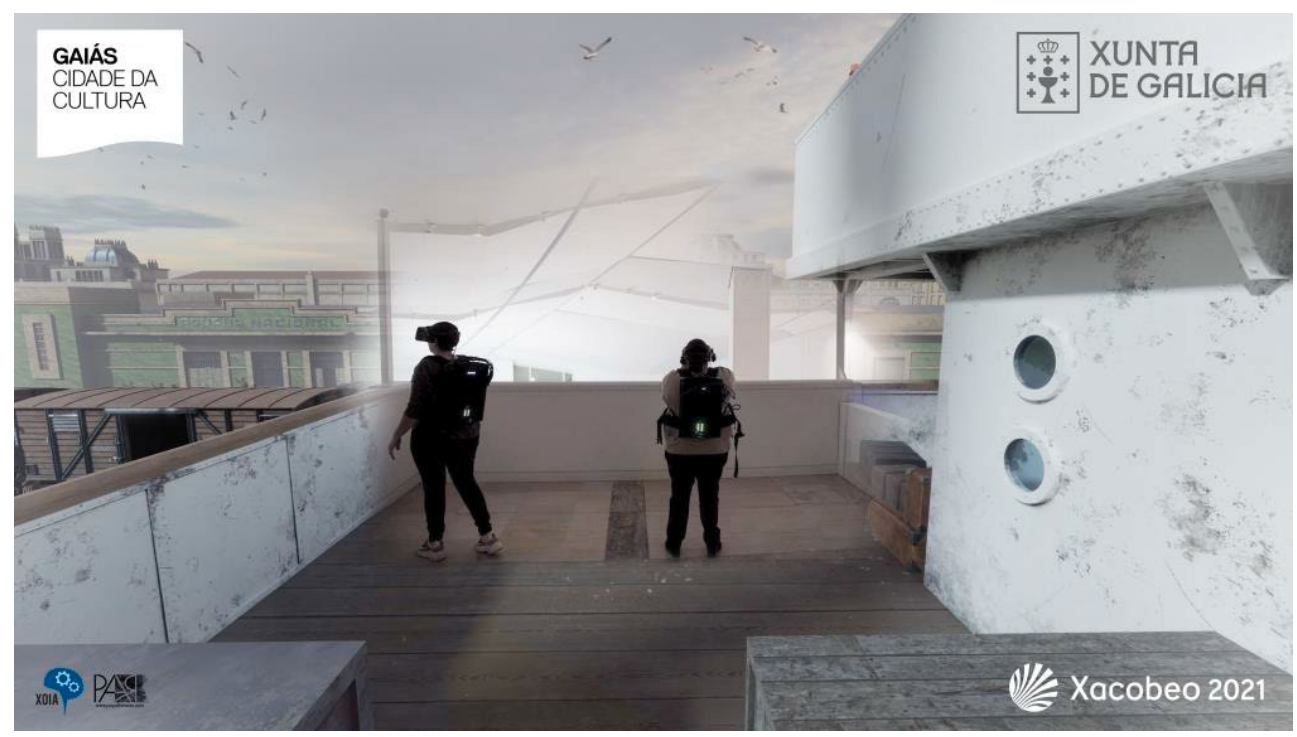

Fig.1. Mochilas y gafas de realidad virtual en una infografía que pretende mostrar la unión entre el espacio real y virtual

En un entorno físico hecho a medida para la experiencia, el usuario puede tocar la reproducción física real de la zona donde se realiza la experiencia y tocar físicamente partes atezadas en el escenario en el propio museo, como barandillas u ojos de buey (fig.2), mientras observa inmersivamente el espacio virtual recreado con las mismas medidas físicas, navegando por el pasado y caminando sobre el propio barco. 


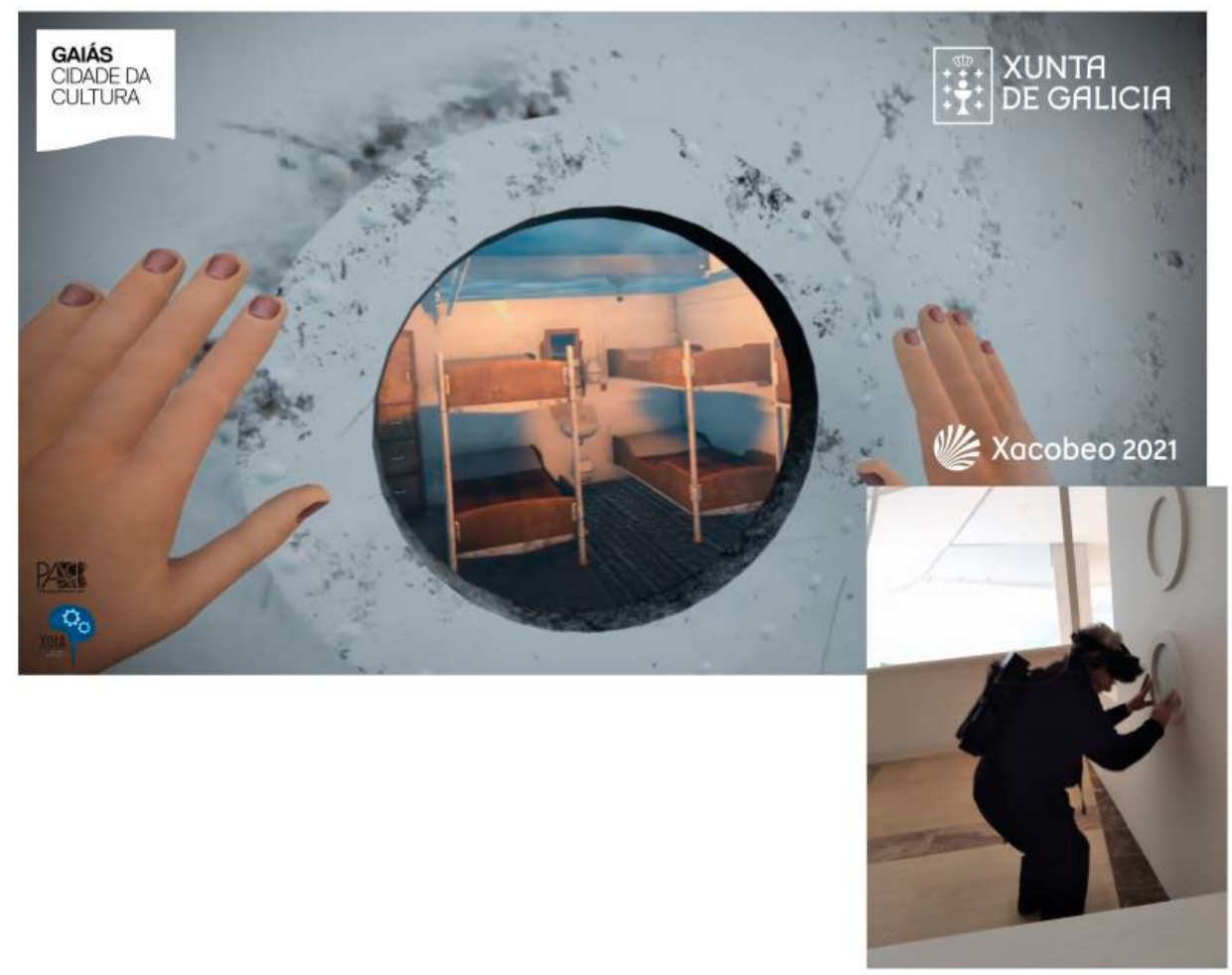

Fig. 2. Un usuario observa el camarote a través de las gafas de Realidad Virtual

El puerto de A Coruña y su gente fueron recreados (PAR Arqueología y Patrimonio Virtual) representados en una escena ambientada en el puerto de A Coruña en 1950, en ella, el usuario se convierte en un emigrante gallego que está a punto de partir del puerto de A Coruña y, desde la borda de un trasatlántico, despide a sus familiares. En esta experiencia se ha optado que la aplicación pueda ser disfrutada hasta por tres personas a la vez, incorporando un novedoso modo multijugador, permitiendo ver a los usuarios en el espacio virtual al resto dentro de sus avatares de época. Capaz de unir el mundo real con el virtual, abre los límites del uso de la realidad virtual en patrimonio explorando nuevas cotas de inmersión que mejora la acción museística además de mostrar la gran accesibilidad que ofrece esta tecnología.

\subsubsection{Realidad Aumentada: O Sebasteion}

En esta experiencia mediante Realidad Aumentada el usuario puede "viajar en el tiempo" y desplazarse al Sebasteion de las Afrodisias en Turquía, recreando el templo en el que se encuentra la referencia al pueblo de los galaicos más distante. Se trata de un monumental templo conmemorativo de la memoria del primer emperador romano, Octavio Augusto. A través de realidad aumentada, las personas visitantes pudieron interactuar con una espectacular réplica a tamaño real de la inscripción y, a través de tabletas, erguir a su alrededor el templo tal y como se conocía finales del Imperio Romano (fig.3). 


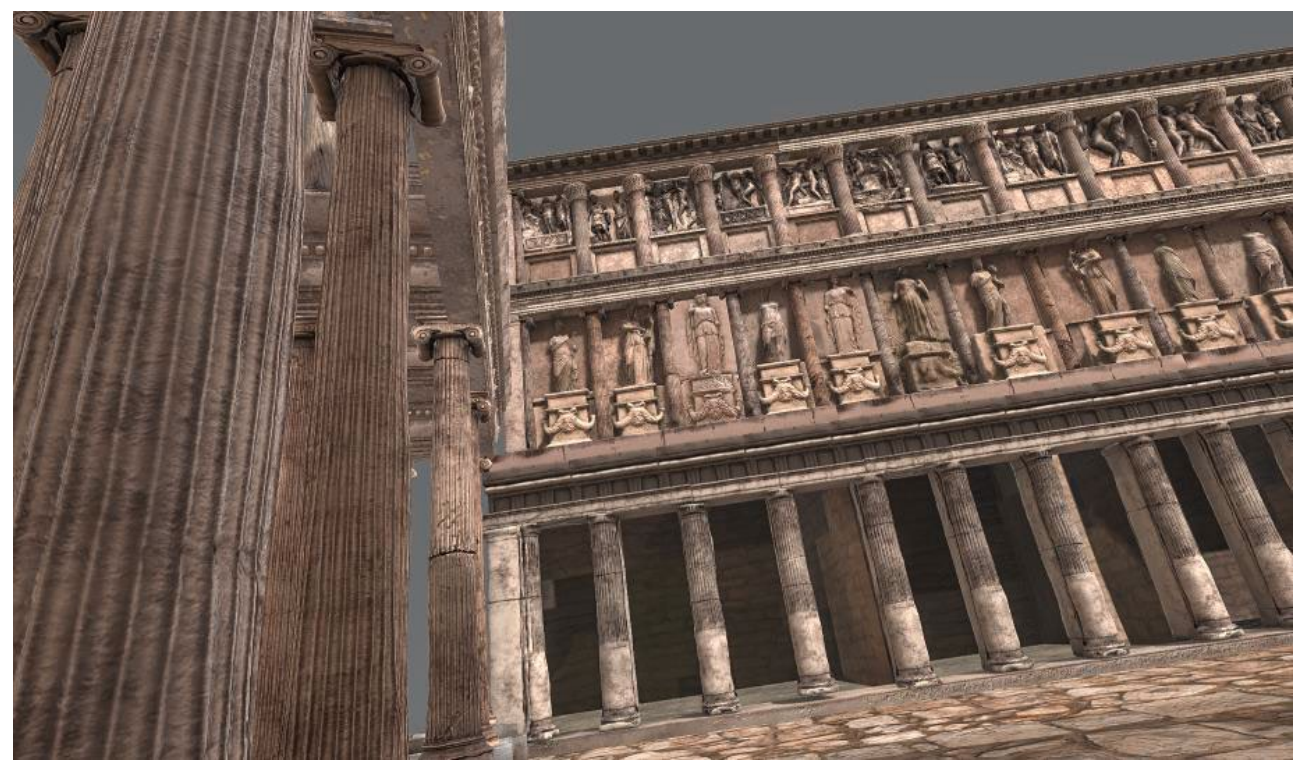

Fig. 3. Reconstrucción hipotética del Sebsteion

Parte de la dificultad técnica consistió en encajar la réplica junto al modelo generado 3D del templo, consiguiéndolo perfectamente mediante una animación en el que se construye el templo en diferentes pisos (fig.4). A su vez, el usuario puede moverse por el espacio habilitado y recorrer el templo mientras porta su Tablet. La aplicación crea conexiones emocionales entre el visitante y la pieza mediante la reconstrucción hipotética del yacimiento reconstruyendo virtualmente tanto a la propia pieza como sus alrededores, trasladándose de forma virtual a la época del Imperio Romano.

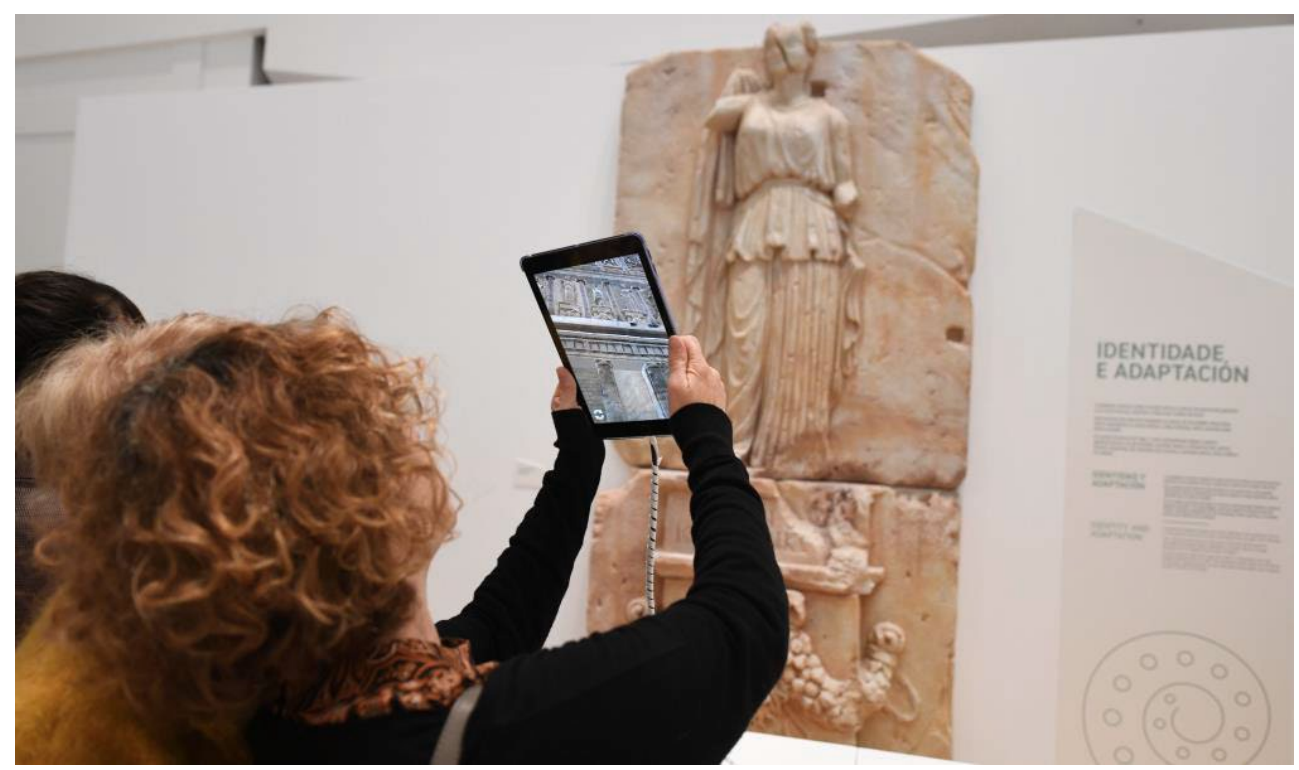

Fig.4. Usuario interactuando con la aplicación de realidad aumentada 


\subsubsection{Sistemas sensoriales y A Mesa das Procedencias}

Otro de los recursos tecnológicos empleados en la muestra por Xoia Extending Reality son los sistemas sensoriales de presencia, que permiten detectar la posición o el interés de los visitantes para desplegar distintos niveles de información. Por una parte, permitieron, sin tocar nada, manipular libros o desplegar mapas que ayudan a comprender el significado y la procedencia de las piezas o desvelan historias guardadas en antiguos códices, narrándolas de forma visual y con recursos tomados de lenguajes próximos al usuario, como el cómic o el grafiti. Para esto, la exposición cuenta con ilustradores como Miguel Peralta, Miguel Robledo o Novenoel. Además, mediante Leap Motion, diferentes proyecciones se despliegan en cuanto los visitantes toman asiento o un recorrido por las diferentes variantes de la lengua gallega y portuguesa a través del mundo completan el despliegue tecnológico con el que la muestra construye su relato sobre los mitos, la historia y la memoria de la identidad gallega a lo largo del tiempo y del mundo.

La mesa de las procedencias consistió en un interactivo con sensores de movimiento que utiliza para mostrar información sobre diversos objetos, yacimientos y culturas en Europa, con objetos físicos de esas culturas traídas de diferentes museos europeos. El usuario puede contemplarlos mientras diversas infografías muestran diferentes aspectos sobre su origen o propiedades. El mismo, sin tocar nada, traslada su mano a los diferentes e individuales sensores de presencia que representan cada uno de los objetos y a su vez desplegar en una pantalla diferente información una vez que los sensores detectan cada expresión de la mano permitiendo al usuario navegar en la información con sencillos paso de delante y atrás (fig.5).

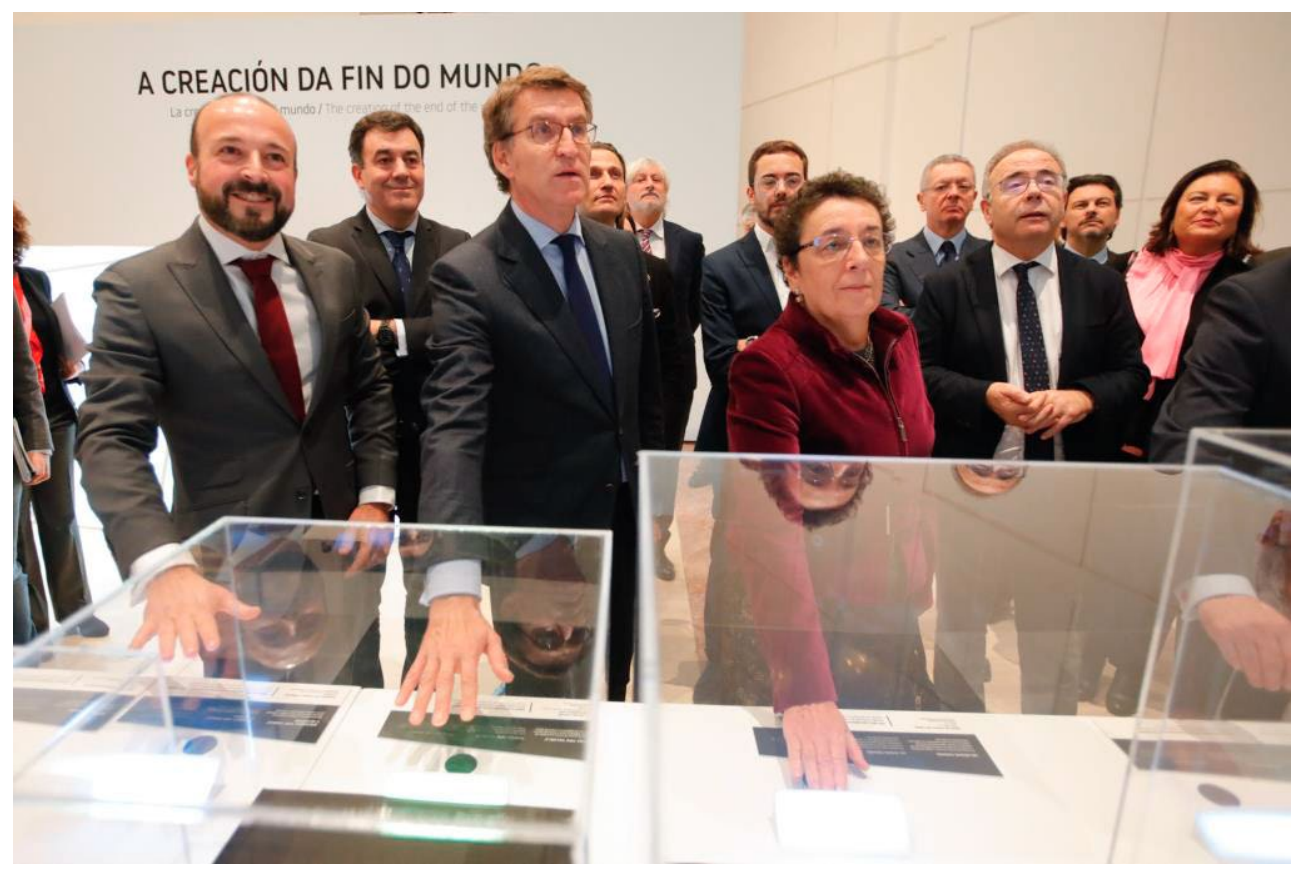

Fig.5. El comisario Manuel Gago (izq.) junto a autoridades en la Mesa de las procedencias 


\subsubsection{Mesa do Camiño}

La aplicación contaba con dos zonas diferentes, una pantalla inferior donde los visitantes interactúan y lanzan las diferentes recreaciones que se visualizan en la pantalla superior. Se ha utilizado la tecnología de Airbars de Neonode, que detecta la presencia de objetos en el espacio, para evitar el uso de pantallas táctiles dedicadas, permitiendo integrar zonas interactivas en una gran superficie. En la pantalla inferior se muestra un mapa de la Europa medieval con las grandes urbes y rutas que tienen relación con el Camino de Santiago de la época, así como los peregrinos que aumentan en número al ir avanzando en la historia.

En la pantalla superior se muestra una recreación en 3D de la Galicia Medieval en forma de cinemática, destacando aquellos lugares y rutas que son importantes en la evolución del Camino de Santiago. Los visitantes pueden escoger qué parte de la historia del Camino de Santiago quieren descubrir, cada pequeña historia aporta nuevos elementos al mapa en 3D de la Galicia medieval. A partir de esta experiencia y gracias a la realidad aumentada se pudo descubrir de una manera divertida la historia del Camino de Santiago medieval en el siglo XII, donde se convirtió en la gran peregrinación que llega hasta nuestros días. Además, el interactuar con una pantalla de grandes dimensiones de manera táctil permite el uso de la mesa de varias personas al mismo tiempo (fig.6).

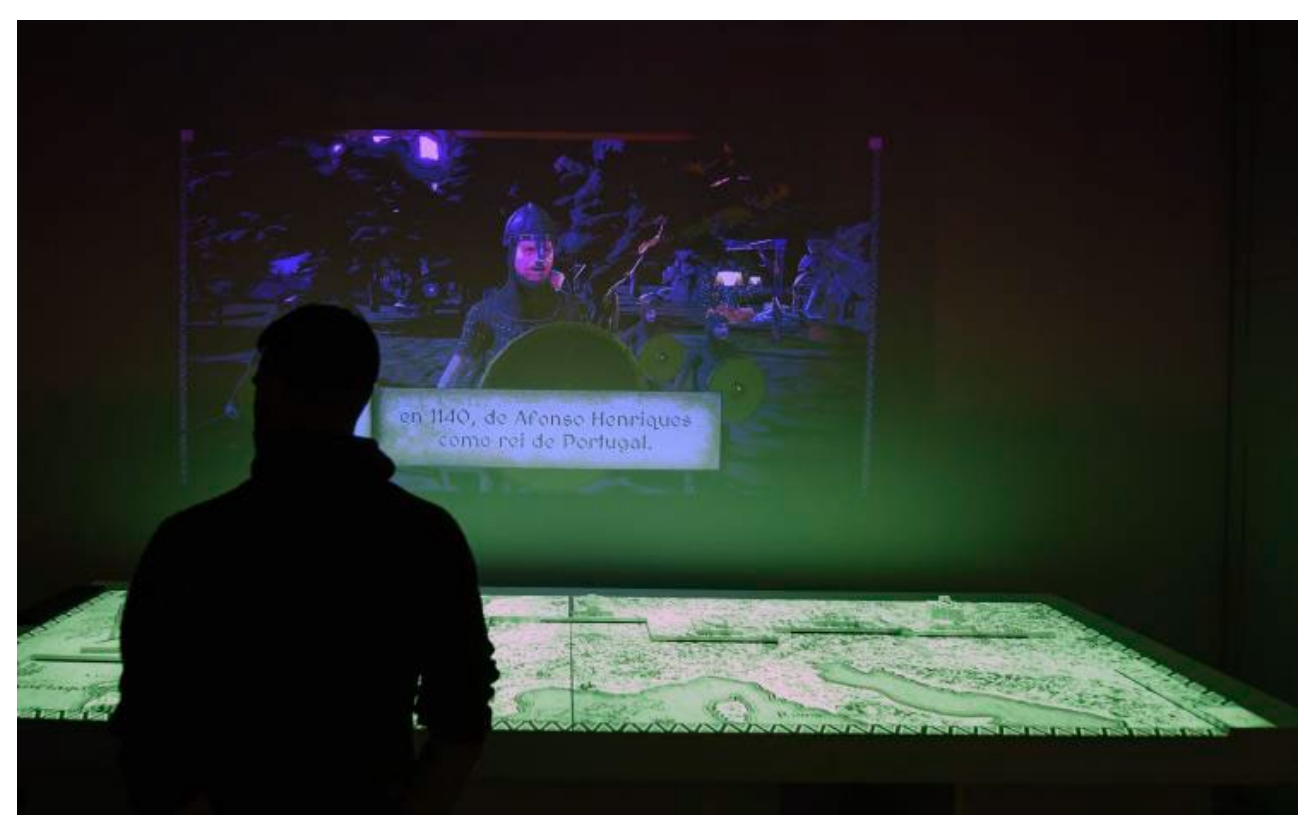

Fig.6. Un usuario interactúa en las proyecciones de A mesa do camino 


\subsection{Exposición "Galicia, de Nós a nós"}

Comisariada por Afonso Vázquez-Monxardín Fernández y Ana Acuña Trabazo, Galicia, de Nós a nós reúne en la Cidade da Cultura más de 300 piezas en la muestra más completa y ambiciosa sobre la Xeración Nós y una de las citas culturales imprescindibles del otoño de 2020, en el que se conmemora el centenario de la publicación de la revista Nós. El Museo Centro Gaiás exhibe obras procedentes de Francia, Alemania y Portugal que ponen el foco en las conexiones internacionales de la revista y de sus impulsores, abriendo una vía para subrayar la dimensión de universalidad y apertura de la cultura gallega. También para destacar el legado del grupo de intelectuales galleguistas que contribuyeron decididamente, en las primeras décadas del siglo XX, al desarrollo, renovación e internacionalización de la lengua y de la cultura gallega. La exposición emplea las nuevas tecnologías como un elemento que ayuda a construir el relato expositivo a través del uso de elementos interactivos, audiovisuales o realidad aumentada. Además, atendiendo a la situación generada por la pandemia de la COVID-19 y para minimizar cualquier riesgo de contagio, todos los elementos interactivos emplean tecnologías sin contacto físico. Para ello se han seguido los siguientes objetivos marcados por la comisión: eliminar de las entrada y la sala en formato papel, sustituyéndolos por su equivalente en formato digital, empleo de tecnologías sin contacto físico para el manejo de las instalaciones interactivas, ajuste del aforo permitido a la normativa vigente en cada momento o el empleo de otras medidas de seguridad de obligado cumplimiento.

\subsubsection{Realidad Aumentada: Principia de Aquis Querquennis}

Esta experiencia, esta vez diseñada para que el usuario pueda descargarla en su teléfono móvil o Tablet debido a la situación de la pandemia COVID - 19, a través de Realidad Aumentada el usuario escanea un target en el suelo sobre el que se reconstruye la estatua del emperador Domiciano y brinda información sobre ella (partes de los restos de dicha escultura están expuestos a escasos metros del target). A través de animaciones 3 , se construye los alrededores que comprende el atrio del principia del campamento romano de Aquis Querquennis (fig.7). El usuario, una vez cargado el escenario, puede cliquear en diferentes objetos en el que se muestra información sobre el yacimiento, las costumbres de quien lo habitaba e información diversa sobre los alrededores, guiados por imágenes de Florentino López Cuevillas, que narra la información cuando el usuario interactúa con el espacio tridimensional. 


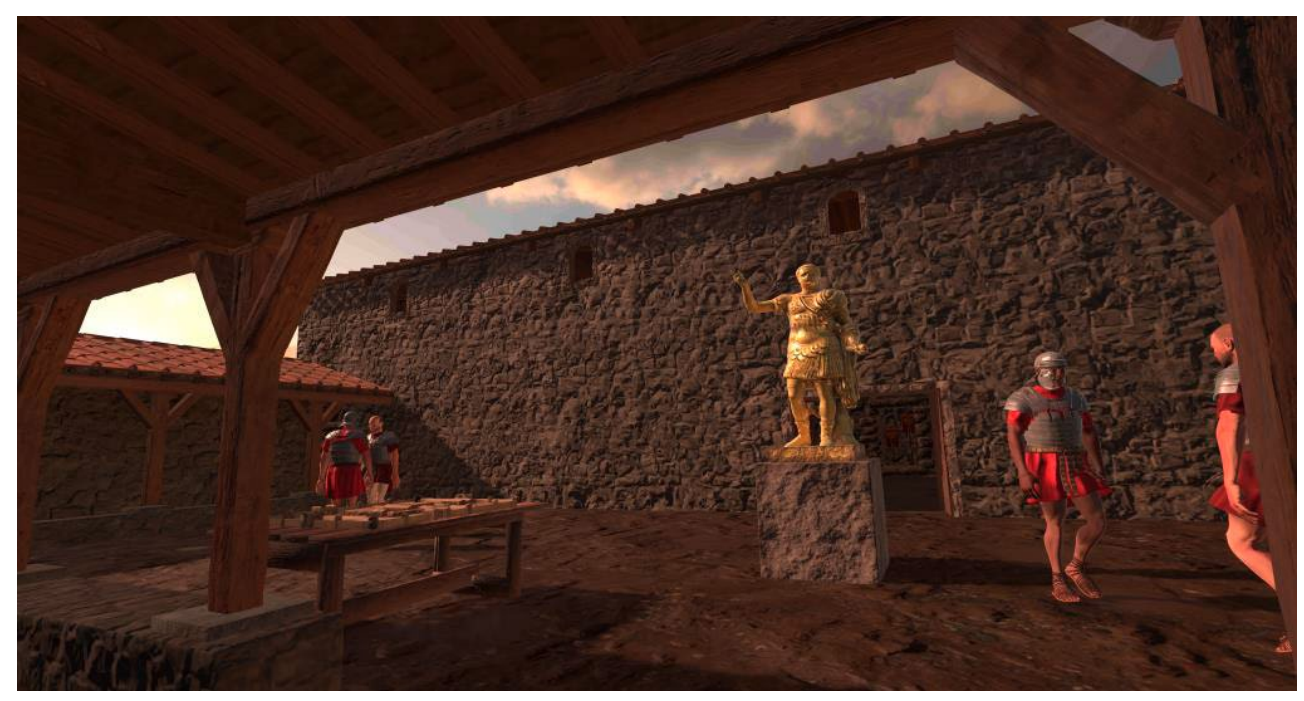

Fig.7. Reconstrucción y recreación del principia de Aquis Querquennis

\subsubsection{Otros interactivos}

Sin tecnología de realidad aumentada pero con un gran componente didáctico importante se realizaron los siguientes interactivos:

$\square$ Interactivo de cartografía: Visualización de ortofotos actuales de las principales ciudades de Galicia (Santiago, Ourense, Pontevedra, A Coruña y Vigo) junto con una comparativa interactiva de distintos mapas realizados por diferentes autores a lo largo del Siglo XIX y XX. El manejo se realiza sin contacto mediante el uso de sensores Leap Motion.

$\square$ Interactivo de portada de la revista Nós: Divulgación de la historia y elementos dela portada de la Revista Nós a lo largo de su tiempo de vida. Utiliza interacción sin contacto mediante el uso de sensores Leap Motion.

Interactivos de catálogo de la revista Nós: Divulgación y visualización de distintos artículos de la revista Nós categorizados en función de distintos autores, temáticas o conexiones con otros países, teniendo un interactivo diferente para cada uno de estos filtros. Utiliza interacción sin contacto mediante el uso de sensores Leap Motion.

\subsection{Fundación María José Jove}

\subsubsection{Realidad Aumentada: ARte Fundación María José Jove}

Esta app disponible en Google Play y Apple Store, sirve de guía dentro de la galería, detectando los cuadros gracias a la realidad aumentada y mostrando información sobre ellos y sus autores. Dicha información va desde descripciones y biografías, pasando por clásicas audio guías y videos de los propios autores, hasta un modo Zoom en el que la aplicación, haciendo uso de la realidad aumentada, mostrará puntos de interés sobre el cuadro para poner 
en valor características o curiosidades del cuadro que pueden obviarse a simple vista. Continuando con el rol de guía, esta app también permite seguir rutas en las que se contextualizan diferentes cuadros de la galería, indicando su localización y facilitando su identificación gracias a la realidad aumentada. La RA permite detectar las diferentes obras de la galería, para acceder de forma directa a su información. Todo el contenido de la aplicación es gestionado a través de una plataforma web. Desde ella puede editarse o ampliarse contenido como las propias obras, su ubicación en la galería, sus descripciones, el audio guías, los videos, los autores, los puntos de interés de las obras o las rutas. Además, gracias al modo Zoom, se puede hacer un uso avanzado de la RA y, sobre la propia obra, se puede interactuar sobre unos puntos de interés que revelan características que pueden quedar ocultas a simple vista. El uso de esta tecnología permite interactuar con la galería de una forma natural e intuitiva mostrando un contenido dinámico y fácilmente gestionable gracias a la plataforma web. Tan fácil como subir una foto de la obra e introducir su información en un formulario web y ya será reconocida por aplicación sin mediación de terceros.

\subsubsection{Realidad Virtual: Museo María José Jove}

Anteriormente se ha mostrado usos de la última tecnología en museos en casos reales. En esta ocasión, el propio museo es digital (fig. 8). Mediante recreación 3D se recrea el museo y el visitante es virtual, es decir, se pone las gafas de Realidad Virtual y puede ver las diferentes obras digitalizadas mediante fotogrametría del museo real, conectando el museo original con el virtual, éste con aspecto diferente al real en una nueva versión sin límites arquitectónicos, creado por arquitectos como si real se tratara y digitalizado por Xoia Extending Reality. Además, mediante un menú interactivo el usuario puede desplazarse por dentro y fuera del museo tele transportándose mientras puede interactuar con cada obra mediante combinaciones con el mando, mostrándose información sobre cada una de las obras. Esta nueva concepción de museo, en donde no se necesita espacio físico real, es una forma de acercar la museología a cualquiera que disponga de unas gafas de RV, el museo se desplaza al usuario y no al revés. Otra de las posibilidades inmersitas de este proyecto, es la de poder visualizar diferentes producciones audiovisuales en pantallas digitales virtuales así como de percibir sonidos y diferente ambientación como sucede en los museos reales, pero con las posibilidades que nos presenta el mundo virtual. 


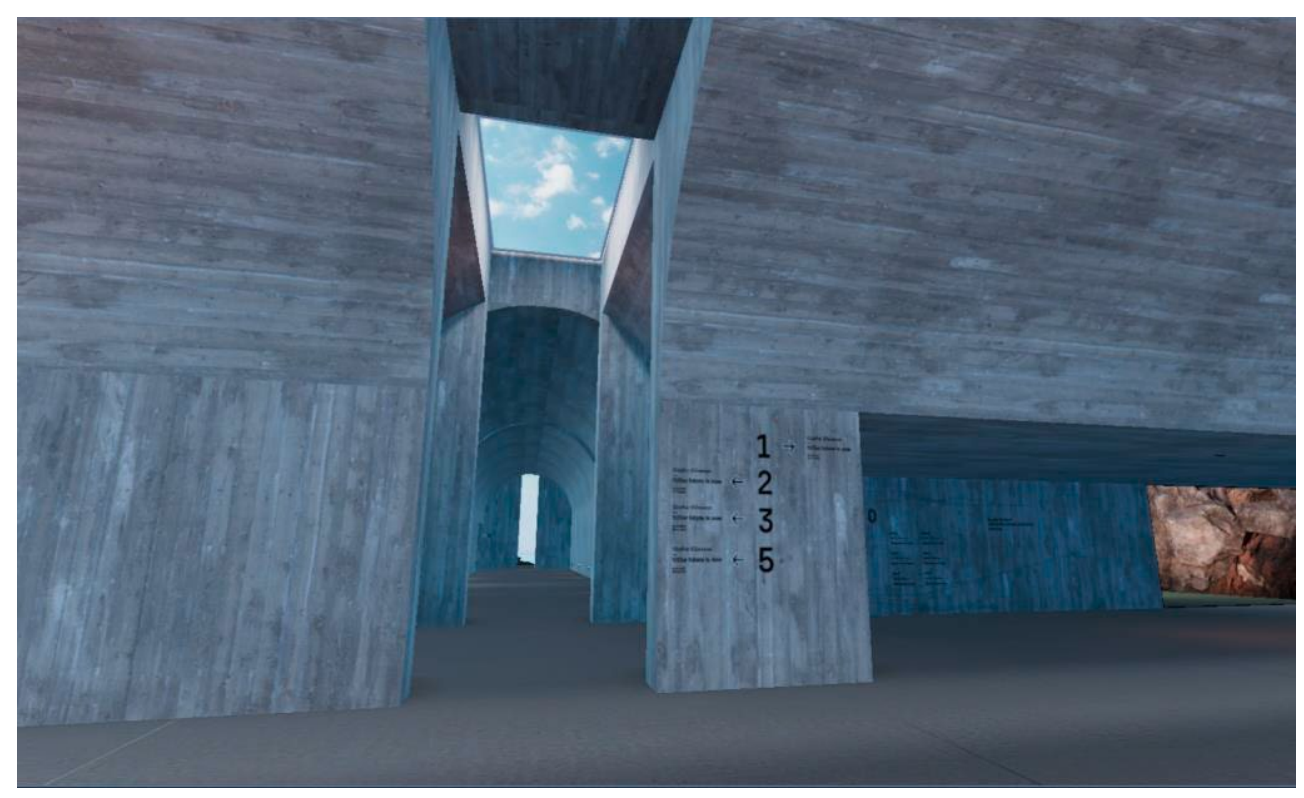

Fig.8. Imagen renderizada del Museo María José Jove

\subsection{Realidad aumentada: Time Trip, una nueva concepción de museo al aire libre}

Las experiencias Time Trip consisten en aplicaciones móviles que usan la realidad aumentada en diferentes targets para mostrar información 3D sobre la historia del lugar en cuestión en peanas físicas cerca de puntos de interés que son recreados en 3D permitiendo ampliar la información de manera visual. Estas aplicaciones de AR sirven de mapa y audio guía turístico digital, llevando al usuario a través de los puntos de interés (POI) gracias a la tecnología GPS y ampliando la información a través de la locución de un personaje animado. En "Time Trip: Viaxando polo Pontedeume medieval", proyecto para el ayuntamiento de Pontedeume, se recrea el aspecto medieval de la villa, desde su aspecto general, hasta de partes más específicas como el Pazo dos Andrade. El usuario puede interactuar cliqueando en diferentes modelos 3D mostrándose información, mientras el personaje narra la información. Disponible en varios idiomas, la locución permite dosificar la información de manera rápida y sin que resulte demasiado pesada como ocurre con el texto (fig. 9). 


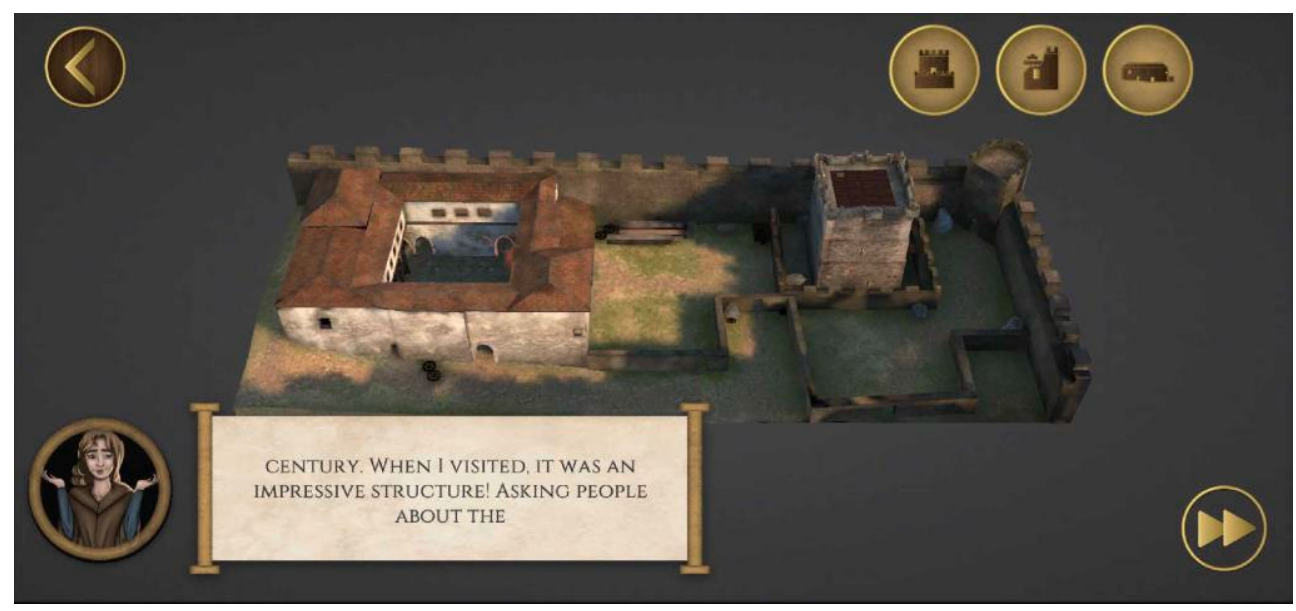

Ilustración 1Fig. 9. Captura de pantalla de la aplicación "Time Trip: Camiñando polo Pontedeume medieval".

De este mismo modo funciona la app "Time Trip: Toralla", realizada para el Ayuntamiento de Vigo, que sirve como guía digital en AR para el museo de Toralla, permitiendo visualizar en Realidad Aumentada el aspecto de la Villa así de cómo se vivía en ese lugar, mostrando recreadas mediante animaciones 3D las diferentes actividades que allí se hacían, como el secado del pescado o los trabajos en las salinas. Las reconstrucciones se han hecho siguiendo una visión histórica, lo que las convierte en una fuente fiable de información. Además, se explica cómo fue evolucionando la villa a través de un sistema de edades donde el usuario puede avanzar y retroceder a voluntad.

En "Time Trip: Visita Molgas" nos trasladamos al rural gallego para mostrar, mediante AR, rutas turísticas digitales que llevan al usuario a través de puntos de interés mediante GPS. Tal como las anteriores, el usuario mediante animaciones en RA recibe información a través de un guía animado. La información de cada POI se muestra en el espacio real, lo cual es innovador como experiencia turísticas y aumenta el interés del usuario además de fomentar el boca a boca. Al mostrarle la ruta al usuario y proporcionarle la información en cada POI se favorece la independencia de este, con esto se apoya a los profesionales del sector turístico diversificando a los visitantes y liberando rutas masivas. En esta app también se muestra la reconstrucción y recreación de un hórreo castreño en el propio yacimiento en el que se muestra información sobre él, y donde el usuario puede interactuar seccionando el hórreo y mostrándose su interior, pudiendo acceder a un botón que muestra el grado de veracidad histórica-arqueológica mediante la superposición de colores que representan la escala Bizancio 2000 y en el que el usuario observa como se ha realizado la interpretación de cada uno de los elementos que componen la reconstrucción del propio hórreo. 


\section{Resultados}

Las experiencias comentadas anteriormente, son fruto de un constante de aprendizaje tanto de innovaciones tecnológicas como de técnicas para llevarlo a cabo. Ante estos cambios tecnológicos fuera o dentro de los espacios museísticos, puede causar miedo el desarrollo de la realidad virtual o aumentada al remplazar en museos que todavía mantienen formas de comunicación más clásicas. Pero al igual que cualquier otra empresa o negocio, deben adaptarse a las nuevas realidades. La apuesta de la RA y RV en museos no es más que una parte más de las diferentes ramas que se puede usar estas tecnologías en crecimiento. Los museos en transición hacia una museología digital, apuestan por la RV y RA para ofrecer una experiencia diferente. Permite agregar vida a los objetos estáticos en el mundo real con sonidos, contenidos visuales e información adicional, expande las ideas más allá de los límites del espacio físico y hasta un teléfono inteligente se puede convertir en un guía personal que no solo puede proporcionar historias textuales, sino que también puede cambiar el tiempo y despertar los objetos. El aumento de la realidad virtual y aumentada brindará nuevas oportunidades y herramientas de narración inmersiva.

Los resultados de los proyectos que han sido comentados, han superado con éxito los objetivos marcados tanto por la organización como cara al público. Un ejemplo es la exposición "Galicia un relato no mundo". La muestra, que cerró sus puertas oficialmente el 12 de abril --aunque no pudo recibir visitas desde el 13 de marzo por motivo del cierre de los museos declarado por la pandemia del COVID-19- congregó un total de 43.035 visitantes, lo que supone una media de 426 visitantes al día, cifra récord en el registro del Museo Centro Gaiás. Una exposición que "abrió la veda" del uso tecnológico de vanguardia en espacios museísticos de Galicia (www.cidadedacultura.gal/, 2020).

La exposición Galicia, de Nós a nós, la segunda gran exposición del Xacobeo 2021, es ejemplo de empleo de las nuevas tecnologías atendiendo a la situación generada por la pandemia del COVID - 19, A través de presencia y movimiento, las personas visitantes puede controlar las diferentes pantallas interactivas de la exposición sin tocar nada y empleando sus dispositivos electrónicos personales en el caso de las recreaciones en realidad aumentada. Una forma de mostrar la versatilidad de las nuevas tecnologías aplicadas en museos, capaz de producir diferentes formas de comunicar atendiendo a las necesidades de cada momento.

En cuanto a las aplicaciones Time Trip, son varios los beneficios que una app de RA de este estilo pueden ofrecer. La adopción de una tecnología novedosa genera atención sobre el pueblo que la adopta y la publicidad que se genera alrededor de la aplicación. El mayor interés sobre los productos que se disponen conlleva un aumento de las visitas al lugar. Además, como se comentó anteriormente, este tipo de experiencias favorece la independencia del usuario, apoyando a los profesionales del sector turístico, diversificando a los visitantes y liberando la carga de personas. 


\section{Conclusiones}

Como se ha expuesto en el presente documento son varias las posibilidades que nos ofrece la Realidad Virtual y el uso de las NTIC, logrando una transición digital de un museo clásico a un museo 4.0 con alta carga digital. El uso de estas tecnologías pretende dar vida a sus colecciones y la tecnología juega un papel muy importante en la experiencia del usuario. En la actualidad, las generaciones jóvenes no visitan los museos tan a menudo como las generaciones anteriores. No es que la cultura, la historia o el arte no sean relevantes para ellos, sino que las experiencias de los museos no coinciden con sus estilos de vida y expectativas. Por ello, usar la realidad virtual o aumentada en los museos es la mejor forma de atraer a la audiencia más joven. Esta aún considera a los museos como lugares estáticos y educativos donde tienen que estar tranquilos y en silencio. Sin embargo, eso ya no tiene que ser así, los museos todavía están abriéndose camino hacia el uso de las nuevas tecnologías y el aumento de este uso digital brindará nuevas oportunidades y herramientas de narración inmersiva para crear el impacto y la experiencia que buscan los jóvenes.

La necesidad de un equipo multidisciplinar, con gran apuesta hacia trabajadores jóvenes y talentosos, serán el prototipo de equipos que formen el nuevo formato de museo. Un museo con gran carga digital, generadores de información y de nuevas vías de comunicación, con gran presencia en las redes sociales e incluso puede proporcionar datos relevantes sobre su uso, mejorando y adaptando la museología 4.0 al usuario. Muestra de ello es lo que se ha conseguido con las exposiciones en el Gaiás, siendo un éxito, con cerca de 420 visitantes al día que supone el récord en el museo desde su apertura, en el que se ha usado gran parte de las tecnologías más innovadoras del momento citadas anteriormente: detectores de presencia, RA, RV, proyecciones audiovisuales,etc. La especialización del equipo ha sido vital para poder trabajar con estas tecnologías, siguiendo una metodología tanto grupal como personal, llevando a cabo proyectos innovadores en grandes exposiciones buscando que estos tengan la aceptación de un público cada vez más tecnológico. Los proyectos mencionados, han sido posible gracias al equipo de Xoia Extending Reality que hace uso de la más variada tecnología para poder subministrar este tipo de herramientas a museos, potenciando su empleo y mostrando nuevas formas de hacer museología, y llevándolo a cabo a su vez, en otras ramas que comprenden la cultura, el turismo o la industria. 


\section{Referencias}

CARO, A. L. (2013) : Nuevas tecnologías para la interpretación y promoción de los recursos turísticos culturales. Pasos, 931-945.

GAIÁS CIDADE DA CULTURA. Programa cultural.

$<$ https://www.cidadedacultura.gal/es/noticias/una-apuesta-por-la-realidad-virtual-yaumentada-para-sumergirse-en-galicia-un-relato-en-el> [Consulta : 1 de marzo 2021]

GRANJA, E. B. (2015) : Realidad Aumentada y su suporte al patrimonio cultural. Universidad Nacional de Chimborazo.

XOIA EXTENDING REALITY. Blog Xoia. $<$ http://www.xoia.es> [Consulta : 1 de marzo 2021] 